\title{
Novel technique for intraductal cholangioscopy-assisted biliary drainage with over-the-wire microcatheter manipulation
}

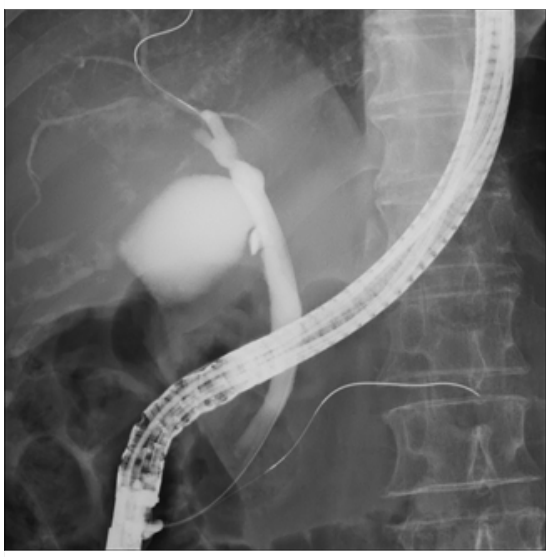

- Fig. 1 Case 1: Endoscopic retrograde cholangiography shows complete obstruction with no flow of contrast into the left intrahepatic bile duct in a patient with hilar cholangiocarcinoma.

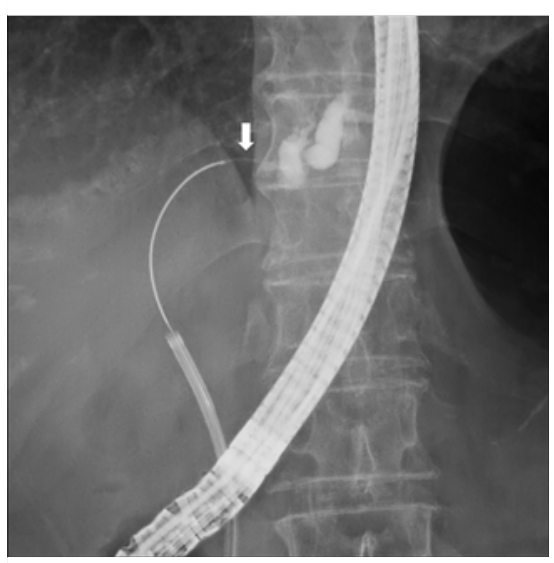

- Fig. 2 A 3-Fr over-the-wire microcatheter (arrow) introduced via intraductal cholangioscopy allowed injection of contrast into the target bile duct.

Cholangioscopy-assisted guidewire placement is reported to be a useful method for endoscopic biliary drainage that is made difficult by complex strictures and obstructions [1-5]. However, the guidewire sometimes becomes misdirected because of the lack of contrastfilled images. With the aim of improving safety and certainty, we present two practical cases that employ a novel tech-

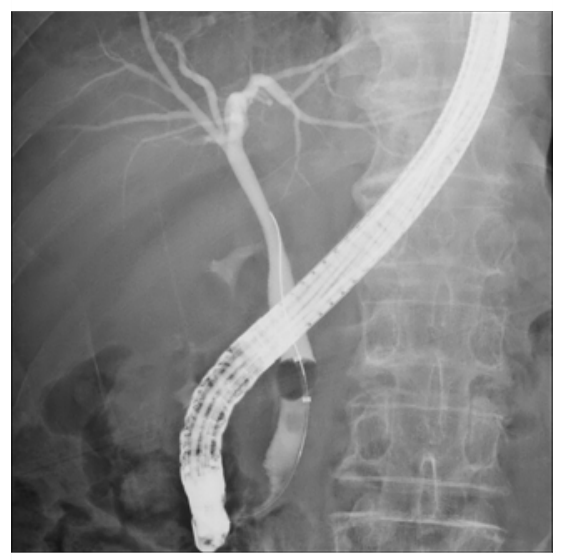

- Fig. 3 Case 2: Endoscopic retrograde cholangiography does not show the orifice of the cystic duct, preventing transcystic guidewire advancement under fluoroscopic imaging in a patient with acute cholecystitis.

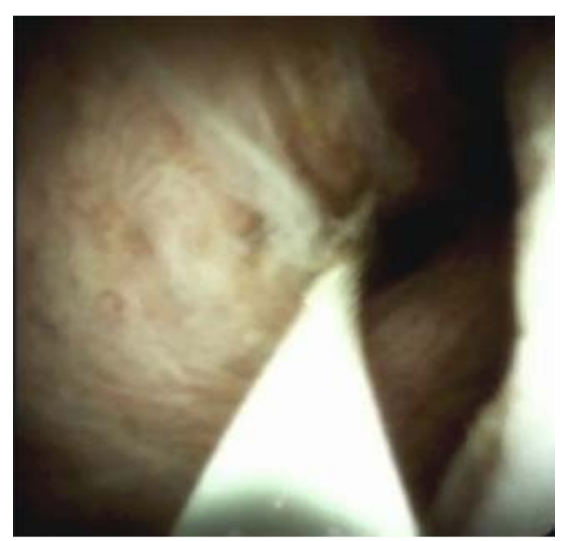

- Fig. 4 Direct cholangioscopy reveals the orifice of the cystic duct and allows insertion of the guidewire with the overthe-wire microcatheter.

nique using an over-the-wire microcatheter through digital intraductal cholangioscopy (IDC) (SpyGlass DS; Boston Scientific, Natick, Massachusetts, USA).

Case 1 involved a 72-year-old woman with hilar cholangiocarcinoma who underwent endoscopic biliary drainage for segmental cholangitis. The cholangiogram showed complete obstruction of the left hepatic duct ( $\triangleright$ Fig. 1). Although

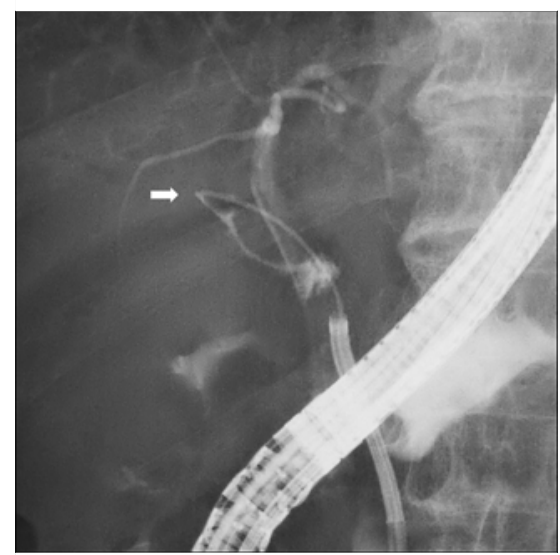

- Fig. 5 Cholangiography on contrast pressure injection via the over-the-wire microcatheter (arrow) shows the extra cavity from the cystic duct, indicating guidewire penetration into the peritoneal cavity.

direct visualization with IDC allowed advancing the 0.025 -inch guidewire over the obstructing tumor in the left hepatic duct, the guidewire lost the pathway to the left intrahepatic bile duct. The 3-Fr outer sheath of a basket catheter (MicroCatch; MTW Endoskopie, Düsseldorf, Germany), which can be inserted into the SpyGlass DS, was introduced as a microcatheter in order to inject contrast medium and assist guidewire manipulation. The contrast-filled image of the left intrahepatic bile duct allowed successful negotiation ( $\triangleright$ Fig.2), followed by replacement of the endoscopic nasobiliary drainage tube ( Video 1 ). Case 2 involved a 79-year-old man with acute cholecystitis. The cholangiogram showed complete obstruction of the cystic duct ( Fig.3), which prevented guidewire advancement under fluoroscopic imaging. The orifice of the cystic duct was visualized using the SpyGlass DS, then the guidewire with a 3-Fr endoscopic nasobiliary drainage tube (Daimon-PTCD set, Hanaco Medical, Saitama, Japan), another microcatheter that may be used through the SpyGlass DS, was advanced into the 


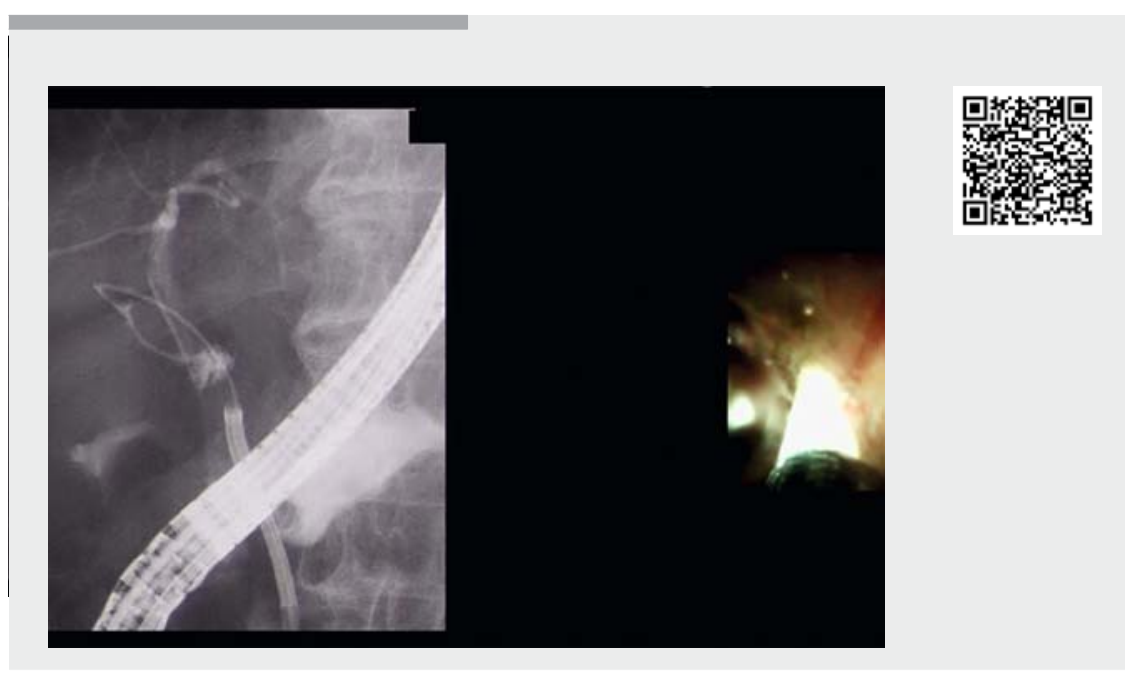

$\checkmark$ Video 1 Novel technique with over-the-wire microcatheter manipulation for SpyGlass DS-assisted selective biliary drainage.

cystic duct ( $\triangleright$ Fig. 4 ). At one point when the guidewire was advanced in an unknown direction, contrast injection through the microcatheter showed clearly that the guidewire had penetrated the peritoneal cavity ( $\triangleright$ Fig.5). The microcatheter also assisted with maneuvering of the guidewire to correct its course, resulting in successful access to the gallbladder, completed by insertion of a plastic stent ( $\vee$ Video 1 ).

Cholangioscopic operation with a microcatheter offers advantages both for obtaining selective contrast-filled images and for delicate manipulation of the guidewire as performed in selective angiographic examinations.

Endoscopy_UCTN_Code_TTT_1AR_2AK

\section{Competing interests}

None
[1] Barkay O, Bucksot L, Sherman S. Endoscopic transpapillary gallbladder drainage with the SpyGlass cholangiopancreatoscopy system. Gastrointest Endosc 2009; 70: 1039- 1040

[2] Shin JU, Lee JK, Kim KM et al. Endoscopic naso-gallbladder drainage by using cholangioscopy for acute cholecystitis combined with cholangitis or choledocholithiasis (with video). Gastrointest Endosc 2012; 76 : $1052-1055$

[3] Bokemeyer A, Gross D, Bruckner M et al. Digital single-operator cholangioscopy: a useful tool for selective guidewire placements across complex biliary strictures. Surg Endosc 2018. doi:10.1007/s00464018-6334-6

[4] Tyberg A, Zerbo S, Kahaleh M et al. Digital cholangioscopy-assisted gallbladder drainage: seeing is accessing. Endoscopy 2015; 47 (Suppl. 01): E417

[5] Yoshida M, Morimoto M, Kato A et al. Recanalization of postoperative biliary disconnection with intraductal cholangioscopy-assisted forceps retrieval of rendezvous guidewire. Endoscopy 2018; 50: E338-E339

Michihiro Yoshida, Akihisa Kato, Kazuki Hayashi, Itaru Naitoh, Katsuyuki Miyabe, Yasuki Hori, Go Asano

Department of Gastroenterology and Metabolism, Nagoya City University Graduate School of Medical Sciences, Nagoya, Japan

\section{Corresponding author}

\section{Kazuki Hayashi, MD}

Department of Gastroenterology and Metabolism, Nagoya City University Graduate School of Medical Sciences,

1 Kawasumi, Mizuho-cho, Mizuho-ku Nagoya 467-8601, Japan

Fax: +81-52-852-0952

khayashi@med.nagoya-cu.ac.jp

\section{ENDOSCOPY E-VIDEOS}

https://eref.thieme.de/e-videos

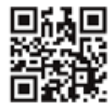

Endoscopy E-Videos is a free access online section, reporting on interesting cases and new

techniques in gastroenterological endoscopy. All papers include a high quality video and all contributions are freely accessible online.

This section has its own submission website at https://mc.manuscriptcentral.com/e-videos 